\title{
A new species of Daldinia (Xylariaceae) from the Argentine subtropical montane forest
}

\author{
Sir EB ${ }^{1}$, Lambert $\mathrm{C}^{2}$, Wendt $\mathrm{L}^{2}$, Hladki $\mathrm{AI}^{3}$, Romero $\mathrm{AI}^{4}$ and Stadler $\mathrm{M}^{2}$ \\ ${ }^{1}$ Fundación Miguel Lillo, CONICET, Institute of Mycology, Miguel Lillo 251, San Miguel de Tucumán 4000, Tucumán, \\ Argentina. \\ ${ }^{2}$ Helmholtz-Zentrum für Infektionsforschung GmbH, Dept. Microbial Drugs, Inhoffenstrasse 7, 38124, Braunschweig, \\ Germany. \\ ${ }^{3}$ Fundacion Miguel Lillo, Institute of Mycology. \\ ${ }^{4}$ Instituto de Micología y Botánica (INMIBO), UBA-CONICET, Depto. Biodiversidad y Biología Experimental, FCEN, \\ Av. Int. Güiraldes 2620, Ciudad Autónoma de Buenos Aires, C1428EHA, Argentina
}

Sir EB, Lambert C, Wendt L, Hladki AI, Romero AI, Stadler M. 2016 - Mycosphere - A new species of Daldinia (Xylariaceae) from the Argentine subtropical montane forest. Mycosphere 7 (9) 13781388, Doi 10.5943/mycosphere/7/9/11

\begin{abstract}
Based on a combination of morphological, molecular phylogenetic and chemotaxonomic evidence, a new species of Daldinia from Northwest of Argentina is described. Daldinia korfii is morphologically related to $D$. placentiformis, but differs in having brown vinaceous, $\mathrm{KOH}-$ extractable pigments and the tissue below the perithecial layer is composed of inconspicuous to conspicuous alternating zones in the new taxon.
\end{abstract}

Key words - Ascomycetes - Yungas - secondary metabolites - Xylariales

\section{Introduction}

Daldinia Ces. \& De Not. (Ascomycota, Xylariales) comprise a group of hypoxyloid Xylariaceae that had been initially erected based on their conspicuous stromata with internal alternating ring zones. Its species are worldwide distributed and principally associated with angiosperms. The genus was first monographed by Child (1932) and Ju et al. (1997). Recently, in the last, comprehensive monograph of Daldinia, Stadler et al. (2014) introduced a modern polythetic concept, involving a combination of morphological, phylogenetic and chemotaxonomic data. In total, including the recently described endophytic, insect associated species D. hawksworthii S. Pažoutová, Šrůtka \& M.Stadler, which is thus far only known from its asexual state (Pažoutová et al. 2013), 48 Daldinia species are currently known from around the world. In Argentina, the genus has hitherto been poorly studied, and the only previous reports go back to Spegazzini $(1902,1909)$ and Hladki (2004).

During a survey Xylariaceae in the "Las Yungas" phytogeographic region (Sir et al. 2016) from northwest Argentina, interesting daldinoid specimens were collected. Our studies based on the modern taxonomic concept revealed that the specimens represent an undescribed species, which we introduce in this paper. 


\section{Materials \& Methods}

The specimens were obtained from different natural reserves from 'Las Yungas" of Jujuy and Salta provinces. The study of sexual state was made according to Stadler et al. (2014). The cultures of the specimen were obtained from multispore isolates as reported by Sir et al. (2016) and the conidiophores branching pattern of the asexual state were classified according to Ju \& Rogers (1996). The color codes of the stromata, extractable pigments and cultures are reported as proposed by Rayner (1970). Reference specimens and cultures are preserved in LIL (Fundación Miguel Lillo, San Miguel de Tucumán, Argentina) and STMA (HZI culture collection, Helmholtz Centre for Infection Research, Braunschweig, Germany).

For chemical analyses, stromata were extracted with methanol (Stadler et al. 2001). Samples were analyzed by analytical HPLC (High performance liquid chromatography) using an Agilent 1260 Infinity Series instrument equipped with a diode array detector and an Electrospray ionisation (ESI)iontrap mass detector (amaZon, Bruker). The instrumental settings were the same as described by Kuhnert et al. (2014a, 2017). Spectra were compared to an internal database with stored signals from standards of known secondary metabolites produced by Xylariaceae from previous work (Bitzer et al. 2007, Stadler et al. 2014). Facesoffungi numbers (Jayasiri et al. 2015) and Index Fungorum (http://www.indexfungorum.org/names/names.asp) numbers are provided.

\section{Molecular phylogenetics}

DNA Isolation, phylogenetic inference and gene amplification of the ITS fragment were performed as described in detail by Otto et al. (2016), while the $\beta$-tubulin fragment was amplified using the protocol of Kuhnert et al. (2014b). Maximum Likelihood trees for both marker genes were calculated based on cured MAFFT (Katoh et al. 2002) alignments and the best fitting substitution models were determined from cured alignments (Gblocks Server by Talavera \& Castresana 2007; with low stringency settings for ITS/ ß-tubulin: Minimum number of sequences for a conserved position: 17/18, minimum number of sequences for a flanking position: 17/18, maximum number of contiguous nonconserved positions: 8 , minimum length of a block: 5 , allowed gap positions: with half) by MEGA modeltest (Tamura et al. 2013). The phylogenetic tree of the ITS region was calculated with PHYML (Guindon et al. 2010); and RAxML (Stamatakis 2014) was used to calculate the phylogenetic tree inferred from $B$-tubulin sequences. Both trees were calculated with 1000 bootstrap replicates (BS). The phylogenetic trees were submitted to TreeBase ( $\beta$-Tubulin tree; TB2:S20192 ITS tree: TB2:S20199) Sources of reference sequences are listed in Table 1.

The phylogenetic tree based on the ITS region of the rDNA (Fig. 1) is composed of 34 sequences, consisting of 23 Hypoxylon, 9 Daldinia, and one Xylaria sequences (Table 1). The backbone of the tree is not statistically supported. The Daldinia sequences form a monophyletic clade, which is supported by a bootstrap value (BS) of $76 \%$. The sequences of the newly described species $D$. korfii cluster together with $D$. placentiformis, while being deliminated from the $D$. eschscholtzii clade (BS value 53\%).

The $\beta$-tubulin tree (Fig. 2) consists of the same genus composition as described above. The backbone is supported partially (BS values of $50 \%$ and above). The Daldinia sequences form a monophyletic clade- and its topology is well supported. EBS476 and EBS473 (D. korfii) cluster together (100\% BS support), while being separated from EBS030 (D. placentiformis). This cluster is differentiated (91\%) from EBS067 (D. korfii). Even though this slight deviation was observed, we find it fully justified to erect a new species, since the morphological and chemotaxonomic data were in accordance in all examined specimens of $D$. korfii.

\section{Taxonomy}

Daldinia korfii Sir \& Lambert, sp. nov. Figs $3-5$ Index Fungorum number: IF552565; Facesoffungi number: FoF 02715 
Table 1 List of used strains for molecular phylogeny. Holotypes (T) and epitypes (ET) are indicated accordingly. EBS codes refer to Sir \& Hladki numbers.

\begin{tabular}{|c|c|c|c|c|c|}
\hline Species & $\begin{array}{l}\text { GenBank } \\
\text { Acc No } \beta- \\
\text { tubulin }\end{array}$ & $\begin{array}{l}\text { GenBank } \\
\text { Acc No ITS }\end{array}$ & $\begin{array}{l}\text { Specimen or } \\
\text { strain ID }\end{array}$ & Origin & Reference \\
\hline $\begin{array}{l}\text { Daldinia } \\
\text { bambusicola }\end{array}$ & AY951688 & KY204022 & CBS 122872 & Thailand & $\begin{array}{l}\text { This study, Hsieh et al. } \\
\text { (2005) }\end{array}$ \\
\hline D. concentrica & КC977274 & AY616683 & CBS 113277 & Germany & $\begin{array}{l}\text { Triebel et al. (2005), } \\
\text { Kuhnert et al. (2014b) }\end{array}$ \\
\hline D. eschscholtzii & КС977266 & JX658484 & MUCL 45435 & Benin & $\begin{array}{l}\text { Stadler et al. (2014), } \\
\text { Kuhnert et al. (2014b) }\end{array}$ \\
\hline D. placentiformis & KY204015 & KY204019 & EBS 030 & Argentina & This study \\
\hline D. korfii sp. nov. & KY204014 & KY204018 & EBS 067 & Argentina & This study \\
\hline D. korfii sp. nov. & KY204016 & KY204020 & EBS 473 & Argentina & This study \\
\hline D. korfii sp. nov. & KY204017 & KY204021 & EBS 476 & Argentina & This study \\
\hline D. placentiformis & КС977278 & AM749921 & MUCL 47603 & Mexico & $\begin{array}{l}\text { Bitzer et al. (2008), } \\
\text { Kuhnert et al. (2014b) }\end{array}$ \\
\hline D. theissenii & KX271247 & KY204023 & CBS 113044 & Argentina & $\begin{array}{l}\text { Stadler et al. (2014), This } \\
\text { study }\end{array}$ \\
\hline $\begin{array}{l}\text { Hypoxylon } \\
\text { calileguense }\end{array}$ & KU604579 & KU604566 & STMA 14059 & Argentina $(\mathrm{T})$ & $\begin{array}{l}\text { Sir et al. 2016, Kuhnert et } \\
\text { al. (2017) }\end{array}$ \\
\hline H. cinnabarinum & AY951709 & JN979409 & BCRB 33810 & Mexico & Hsieh et al. (2005) \\
\hline H. erythrostroma & КС977296 & КС968910 & MUCL 53759 & Martinique & Kuhnert et al. (2014b) \\
\hline H. fendleri & AY951718 & JN979418 & BCRB 34064 & Taiwan & Hsieh et al. (2005) \\
\hline H. fragiforme & - & AY616690 & CBS 114745 & Germany & Triebel et al. (2005) \\
\hline H. fragiforme & AY951719 & - & BCRC 34065 & France & Hsieh et al. (2005) \\
\hline $\begin{array}{l}\text { H. fulvo- } \\
\text { sulphureum }\end{array}$ & KP401584 & KP401576 & $\begin{array}{l}\text { MFLUCC 13- } \\
0589\end{array}$ & Thailand $(\mathrm{T})$ & Sir et al. (2015) \\
\hline H. fuscum & AY951723 & JN979423 & BCRC 34069 & Taiwan & Hsieh et al. (2005) \\
\hline H. griseobrunneum & КC977281 & KC968928 & $\begin{array}{l}\text { MUCL } \\
53310, \text { CBS } \\
129346\end{array}$ & Guadeloupe & Kuhnert et al. (2014) \\
\hline H. haematostroma & KU159527 & KU604596 & STMA 14043 & Argentina & Sir et al. (2016) \\
\hline H. hypomiltum & КC977298 & КС968914 & MUCL 53312 & Guadeloupe & Kuhnert et al. (2014b) \\
\hline H. investiens & KC977270 & KC968925 & CBS118183 & Malaysia & $\begin{array}{l}\text { Bitzer et al. (2008), } \\
\text { Kuhnert et al. (2014b) }\end{array}$ \\
\hline H. laminosum & KC977292 & КС968934 & $\begin{array}{l}\text { MUCL } \\
53305, \\
\text { CBS129032 }\end{array}$ & $\begin{array}{l}\text { Martinique } \\
(\mathrm{T})\end{array}$ & Kuhnert et al. (2014b) \\
\hline H. lateripigmentum & KC977290 & КС968933 & $\begin{array}{l}\text { MUCL } \\
53304, \text { CBS } \\
129031\end{array}$ & $\begin{array}{l}\text { Martinique } \\
\text { (T) }\end{array}$ & Kuhnert et al. (2014) \\
\hline H. lenormandii & KM610305 & KM610289 & BCC 71961 & Thailand & Kuhnert et al. (2015) \\
\hline H. lienhwacheense & KU159522 & KU604558 & $\begin{array}{l}\text { MFLUCC 14- } \\
1231\end{array}$ & Thailand & Sir et al. (2016) \\
\hline H. lilloi & KU159537 & KU604574 & STMA 14142 & Argentina (T) & Kuhnert et al. (2017) \\
\hline H. lividipigmentum & KU159529 & KU604567 & STMA 14044 & Argentina & Sir et al. (2016) \\
\hline H. monticulosum & KP401578 & KP401585 & $\begin{array}{l}\text { MFLUCC 13- } \\
\text { 0593, BCC } \\
71965\end{array}$ & Thailand & Sir et al. (2015) \\
\hline H. ochraceum & KC977300 & KC968937 & MUCL 54625 & $\begin{array}{l}\text { Martinique } \\
\text { (ET) }\end{array}$ & Kuhnert et al. (2014b) \\
\hline H. perforatum & КС977299 & KC968936 & MUCL 54174 & Japan & Kuhnert et al. (2014b) \\
\hline H. pulicicidum & JX183072 & JX183075 & $\begin{array}{l}\text { MUCL } \\
49879, \text { CBS } \\
122622\end{array}$ & $\begin{array}{l}\text { Martinique } \\
\text { (T) }\end{array}$ & Bills et al. (2012) \\
\hline
\end{tabular}




\begin{tabular}{|c|c|c|c|c|c|}
\hline H. rickii & КС977288 & КС968932 & $\begin{array}{l}\text { MUCL } \\
53309, \text { CBS } \\
129345\end{array}$ & $\begin{array}{l}\text { Martinique } \\
\text { (ET) }\end{array}$ & Kuhnert et al. (2014b) \\
\hline H. spegazzinianum & KC604582 & KU604573 & STMA 14082 & Argentina $(\mathrm{T})$ & Kuhnert et al. (2017) \\
\hline Xylaria hypoxylon & KX271279 & KY204024 & CBS 122620 & Sweden & $\begin{array}{l}\text { This study, Stadler et al. } \\
\text { 2014a) }\end{array}$ \\
\hline
\end{tabular}

Holotype - Argentina, Jujuy Province, Dept. Ledesma, Calilegua National Park, "La Lagunita" trail, 11 May 2012; Sir \& Hladki 067 (LIL, ex-type culture STMA 16158, GenBank Acc. No: ITS - KY204018, B-tubulin - KY204014).

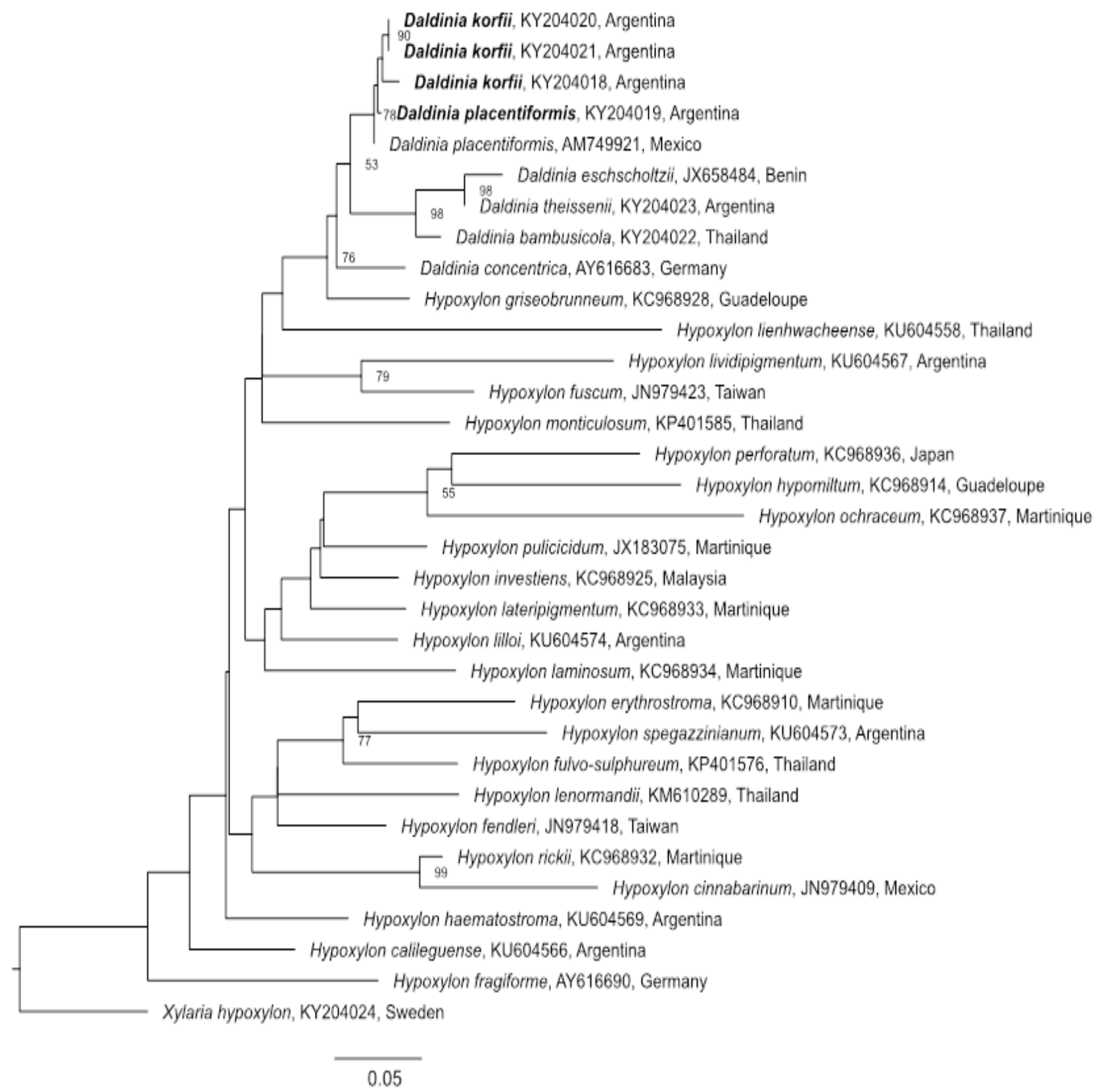

Fig. 1 - Phylogenetic tree (PHYML, substitution model K80+G, BS10000; $\log \mathrm{L}=-5372.23$ ) of selected Daldinia and Hypoxylon species, inferred from the ITS region. Bootstrap support values of $50 \%$ and above are indicated at the respective nodes. Daldinia species discussed in this paper are highlighted in bold letters. 


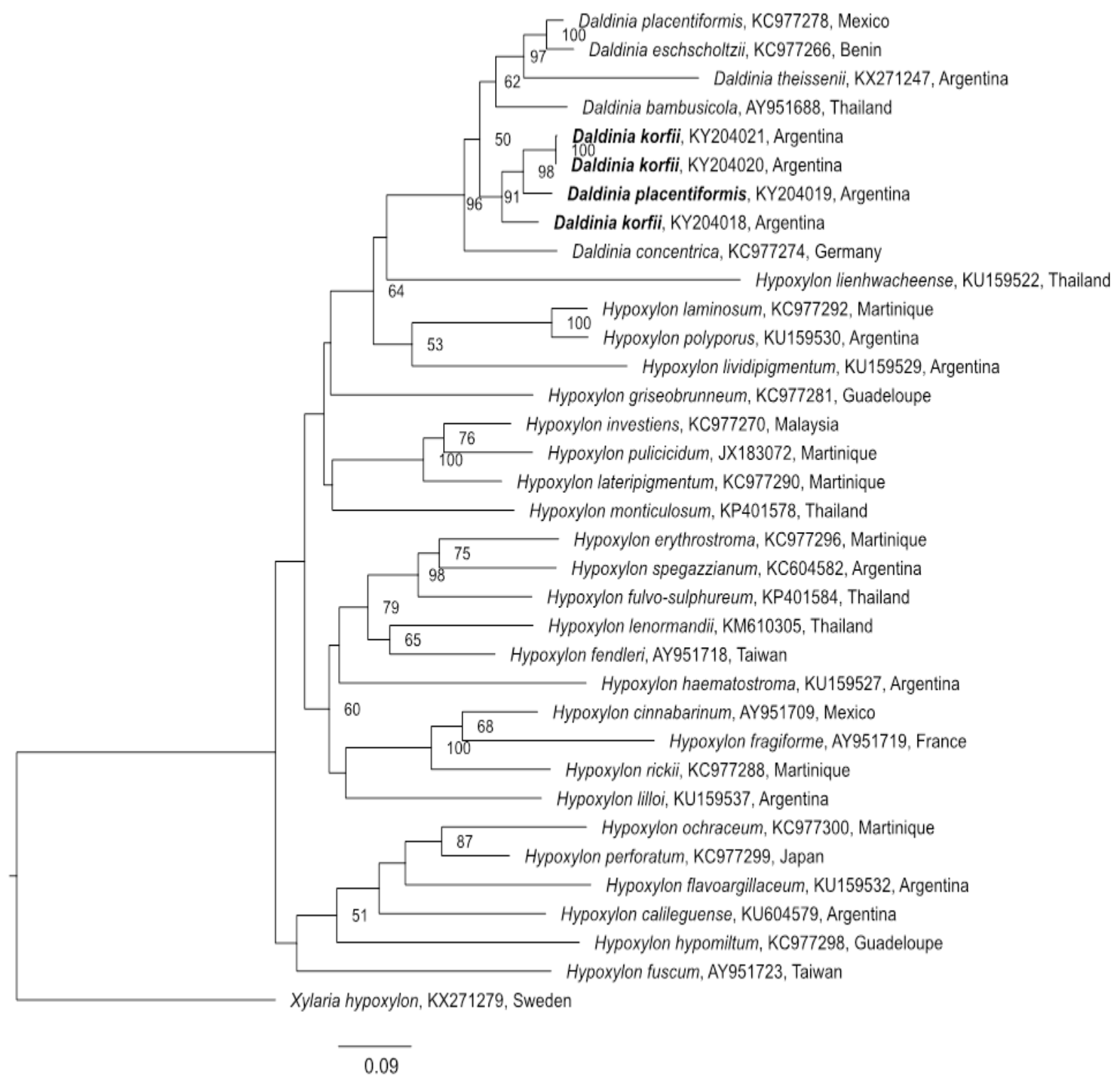

Fig. 2 - Phylogenetic tree (RAxML, GTR+G+I, BS1000; $\log \mathrm{L}=-13927.95)$ of selected Daldinia and Hypoxylon species, inferred from the $\beta$-tubulin gene region Bootstrap support values of $50 \%$ and above are indicated at the respective nodes. Daldinia species discussed in this paper are highlighted in bold letters.

Etymology - In honour of Dr. Richard P. Korf, one of the leading mycological taxonomists of the $20^{\text {th }}$ century.

On dead angiosperm wood. Sexual morph - Stromata hemispherical, pulvinate or peltate, the base broadly attached to the substrate or constricted generally, coalescent (Fig. 3a-d), with inconspicuous to conspicuous perithecial mounds (Fig. 3k), 8-55 mm long $\times 8-30 \mathrm{~mm}$ broad $\times 5-13$ mm thick. Surface Brown Vinaceous (84), pruinose, dark red-orange granules forming a thin crust above perithecia, with Brown Vinaceous (84) to Dark Vinaceous (82) KOH-extractable pigments (Fig. 1e); the tissue between perithecia pale brown to brown; the tissue below the perithecia with layer composed of alternating zones (Fig. 3g), darker zones gray-brown, pithy, 0.4-1.2 mm thick, formed by cylindrical cells oriented vertically (Fig. 3h and i); the dark-brown zone, pithy, 0.7-1.6 $\mathrm{mm}$ thick, forming by cylindrical cells oriented horizontally (Fig. $3 \mathrm{~h}$ and j). Perithecia lanceolate (Fig. 11), 1.1-1.8 $\times 0.3-0.7 \mathrm{~mm}$, ostioles inconspicuous, punctiform to umbilicate (Fig. 3k). Asci 8spored, cylindrical, 169-311 $\mu \mathrm{m}$ total length, the spore-bearing parts $62-100 \times 7-11 \mu \mathrm{m}$, the stipes 72.5-228 $\mu \mathrm{m}$ long. (Fig. 4a), with amyloid, discoid apical apparatus 
(Fig. 2, c), 0.5-1 $\times 1.7-3 \mu \mathrm{m}$. Ascospores brown to dark brown, ellipsoid-inequilateral with narrow rounded ends (Fig. 4b), (10.3) 11-14 (16) × (4.8) 5.2-6.2 (7) $\mu \mathrm{m},(\mathrm{N}=181, \mathrm{Me}=12.5 \times 5.8 \mu \mathrm{m})$, with straight germ slit spore-length on convex side (Fig. 4f); perispore dehiscent in $10 \% \mathrm{KOH}$, smooth (Fig. 4d-e); epispore smooth. Asexual morph - Conidiophores with virgariella-like to nodulisporium-like branching pattern as defined in Ju and Rogers (1996) (Fig. 4j-1), erect, 200$300 \mu \mathrm{m}$ high, melanized main axis in some cases, hyaline to pale green, smooth to roughened. Conidiogenous cells cylindrical, hyaline to pale green, smooth to finely roughened (Fig. 4m), 11-26 $\times 2-3.5 \mu \mathrm{m}$. Conidia hyaline, smooth, ellipsoid, 5-8 × 2-4 $\mu \mathrm{m}$ (Fig. 4n).

Cultures - Colonies on OA covering Petri dish in 3 weeks, at first whitish becoming Olivaceous Gray (121) velvety to felty, azonate with entire margin (Fig. 4g), reverse, Dull Green (21), Olivaceous (48); no conidiogenous structures were observed. Colonies on YMG covering Petri dish in 4 weeks at first whitish becoming Olivaceous Gray (121) velvety to felty, azonate with entire margin (Fig. 4g-h), reverse Olivaceous Black (106). Sporulation regions Dull Green (70).

Secondary metabolites - The stromata contain BNT (binaphthalene tetrol), concentricol B and different cytochalasins as main metabolites (Fig. 5).

Host - Native angiosperms from "Las Yungas"

Additional material examined - Argentina, Jujuy province, Dept. Ledesma, Calilegua National Park, "La Lagunita" trail, 11 May 2012; Sir \& Hladki 066 (LIL); "Sendero del Cielo", 12 May 12, Sir \& Hladki 032 (LIL); "La Lagunita" trail, 26 April 2014, Sir \& Hladki 631 (LIL). Dept. Santa Bárbara, Las Lancitas Provincial Reserve, 13 May 2012; Sir \& Hladki 288 (LIL), 27 April 2014; Sir \& Hladki 683, 684 (LIL). Salta province, Depto. Anta, El Rey National Park, "El Chorro de los Loros" trail, 29 April 2014, Sir \& Hladki 710, 723 (LIL). Dept. Gral. José de San Martin, near to Acambuco Provincial Reserve, 23 April 2014, Sir \& Hladki 547 (LIL). Santa Victoria, El Nogalar de los Toldos National Reserve, 26 June 2013; Sir \& Hladki 473 (cultured, code STMA 14089); same locality, Sir \& Hladki 476 (cultured, code STMA 14092 LIL).

Notes - Daldinia korfii can be identified by its massive subperithecial tissue with inconspicuous to conspicuous concentric zones and $\mathrm{KOH}$-extractable pigments brown vinaceous. Our microscopic study revealed that the concentric zones correspond to different orientations of the hyphae (Fig. 1e-h).

Daldinia placentiformis (Berk. M.A. Curtis) Theiss. appears to be closely related to the new species but differs in having green olivaceous $\mathrm{KOH}$-extractable pigments and by the absence of concentric zones in its subperithecial tissue. In addition, its stromata contain daldinone A as major secondary metabolite (Hellwig et al. 2005). The discovery of the new species provides additional evidence that some members of the " $H$. placentiforme complex" sensu Ju and Rogers (1996) are related to Daldinia, while other species with highly similar stromatal macromorphology such as $H$. polyporus (Starb.) Y.M. Ju \& J.D. Rogers may represent a different phylogenetic lineage in the hypoxyloid Xylariaceae.

Bitzer et al. (2008) reported a somewhat aberrant specimen matching the features of $H$. placentiforme sensu Ju \& Rogers (1996) from South Africa (MUCL 47603) with larger amounts of BNT (and purple stromatal pigments as verified in the current study). We noted that the conidiogenous cells and conidia of this material have similar dimensions to those of the new taxon. However, their ascospores are shorter [10-12.5(-13.5) $\times 6-7$ vs $(10.3) 11-14(16) \times(4.8) 5.2-6.2$ (7) $\mu \mathrm{m}$ ] (see Table 2). Moreover, D. korfii contains cytochalasins and concentricol B as stromatal metabolites. These are chemotaxonomic marker molecules for D. concentrica (Bolton: Fr.) Ces. \& De Not., D. eschscholtzii (Ehrenb.: Fr.) Rehm and some members of the D. eschscholtzii group (Quang et al 2002; Stadler et al. 2014) but they have never been found in typical D. placentiformis. The South African fungus resembling D. placentiformis, which may warrant erection of another new species as more collections become available, was reported to contain only BNT and further metabolites with similar UV/Vis spectra that presumably constitute other binaphthyl derivatives, but neither cytochalasins nor concentricols were detected in its stromata (cf., Bitzer et al. 2008). 

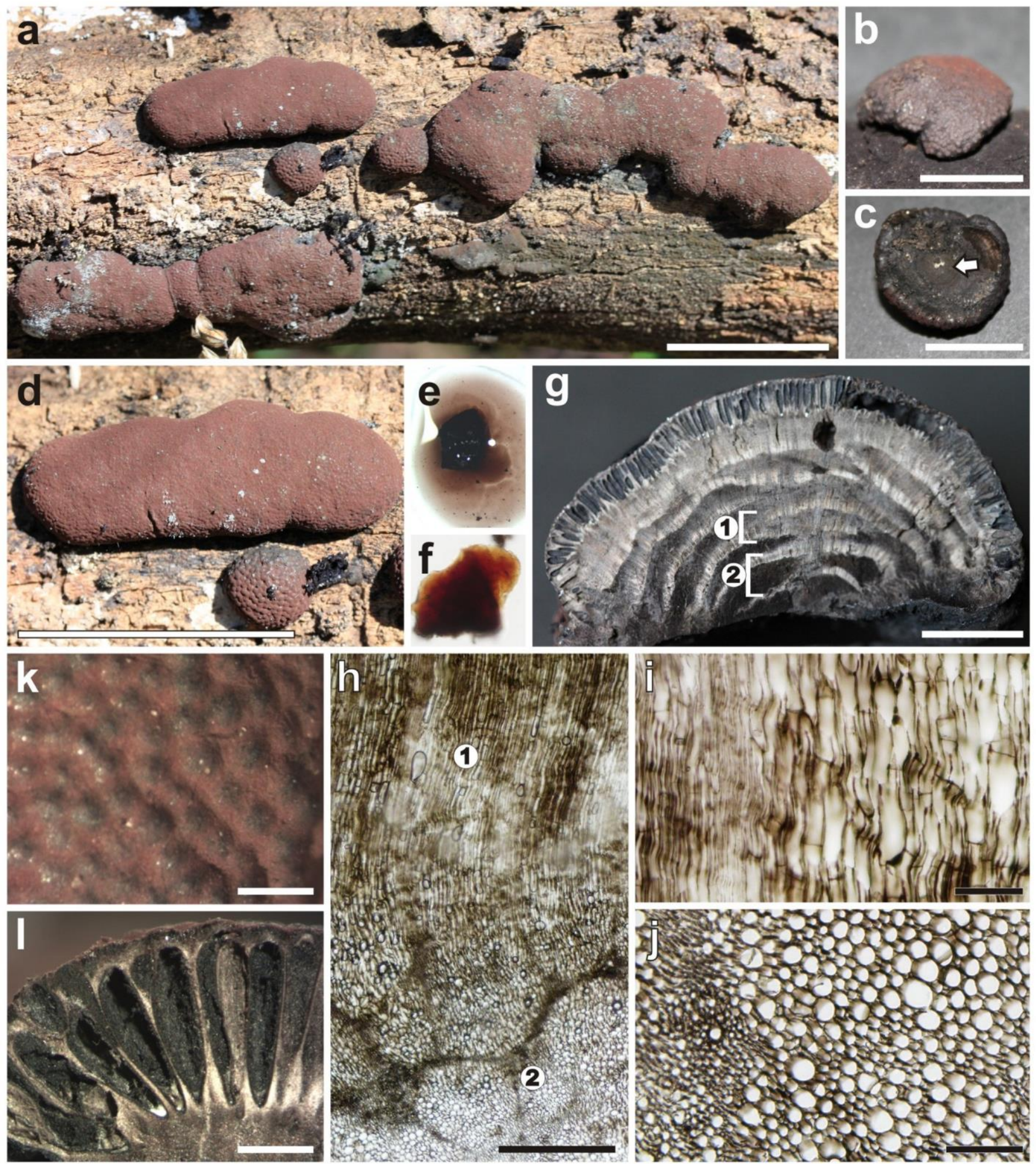

Fig. 3 - Daldinia korfii (Holotype) a, b, d. stromatal habit. c. stromata showing ventral insertion point (white arrow). e. $\mathrm{KOH}$-extractable pigments in $10 \% \mathrm{KOH}$. f. stromatic granules. g. stroma in longitudinal section showing internal concentric zones ( 1 and 2). h. cells of internal concentric zones, in 1 cells oriented vertically, in 2 cells oriented horizontally. i. detail of cells oriented vertically. j. detail of cells oriented horizontally. $\mathrm{k}$. stromatal surface 1 . perithecia in longitudinal section. Scale bars: $a, d=3 \mathrm{~cm} . \mathrm{b}, \mathrm{c}, \mathrm{g}=0.5 \mathrm{~mm} . \mathrm{h}=40 \mu \mathrm{m} . \mathrm{i}, \mathrm{j}=10 \mu \mathrm{m} . \mathrm{k}, \mathrm{l}=1 \mathrm{~mm}$. 


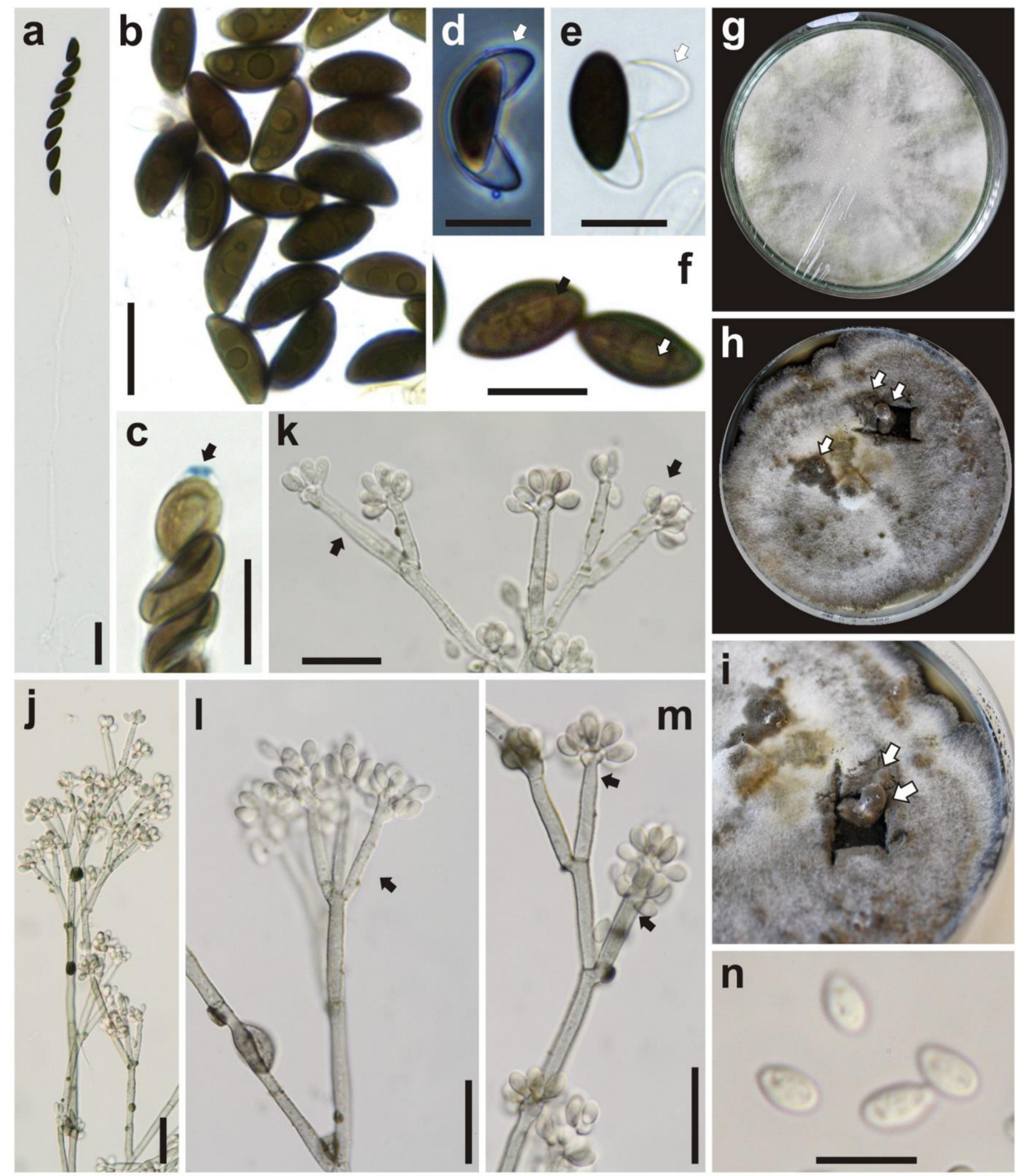

Fig. 4 -Daldinia korfii. a. asci. b. ascospores. c. amyloid apical apparatus (black arrow). d. ascospore with dehiscent perispore in polarized light (white arrow). e. ascospore in $\mathrm{KOH}$ showing dehiscent perispore (withe arrow). f. ascospores showing germ slit (arrows). g. culture on OA after 3 weeks. h, i. culture on YMG after 4 weeks, showing areas of production of immature stromata (white arrows). j. anamorphs showing conidiophores with virgariella-like to nodulisporium-like branching patterns. k. virgariella-like branching pattern (arrows). 1. nodulisporium-like branching pattern (arrow). m. conidiogenous cells (arrows). $n$ conidia. Scale bars: $a, j-m=20 \mu \mathrm{m}$. b-f, $n=10 \mu \mathrm{m}$. 
Table 2 Comparison of Daldinia korfii and D. placentiformis

\begin{tabular}{|c|c|c|c|c|}
\hline & $\begin{array}{l}\text { Ascospores } \\
\text { size }(\mu \mathrm{m})\end{array}$ & $\begin{array}{l}\text { KOH- } \\
\text { extractable } \\
\text { pigments } \\
\end{array}$ & $\begin{array}{l}\text { Anamorph } \\
(\mathrm{Cc} \text { and } \mathrm{Co} \text { in } \mu \mathrm{m})\end{array}$ & References \\
\hline D. placentiformis & $14.5-16 \times 6.5-7$ & Dull Green* & $\begin{array}{l}\text { nodulisporium-like } \\
\text { Cc: } 11-20 \times 2-3.5 \\
\text { Co:4.5-6.5 × } 2.8-3.5\end{array}$ & $\begin{array}{l}\text { Stadler et al. } \\
(2014)\end{array}$ \\
\hline $\begin{array}{l}\text { D. cf. placentiformis } \\
\text { (MUCL 47603) }\end{array}$ & $10-12.5(-13.5) \times 6-7$ & Purple & $\begin{array}{l}\text { nodulisporium-like } \\
\text { Cc: } 14-25 \times 2.5-3.5 \\
\text { Co: } 5.5-7.5 \times 3.5-4\end{array}$ & $\begin{array}{l}\text { Bitzer et al. } \\
(2008)\end{array}$ \\
\hline D. korfii & $\begin{array}{l}(10.3) 11.0-14.0(16.0) \\
\times(4.8) 5.2-6.2(7.0)\end{array}$ & $\begin{array}{l}\text { Brown } \\
\text { Vinaceous* }\end{array}$ & $\begin{array}{l}\text { nodulisporium-like } \\
\text { Cc: } 11-26 \times 2-3.5 \\
\text { Co: } 5-8 \times 2-4\end{array}$ & This study \\
\hline
\end{tabular}

Cc - Conidiogenous cells. Co - Conidia. * - following Rayner (1970)

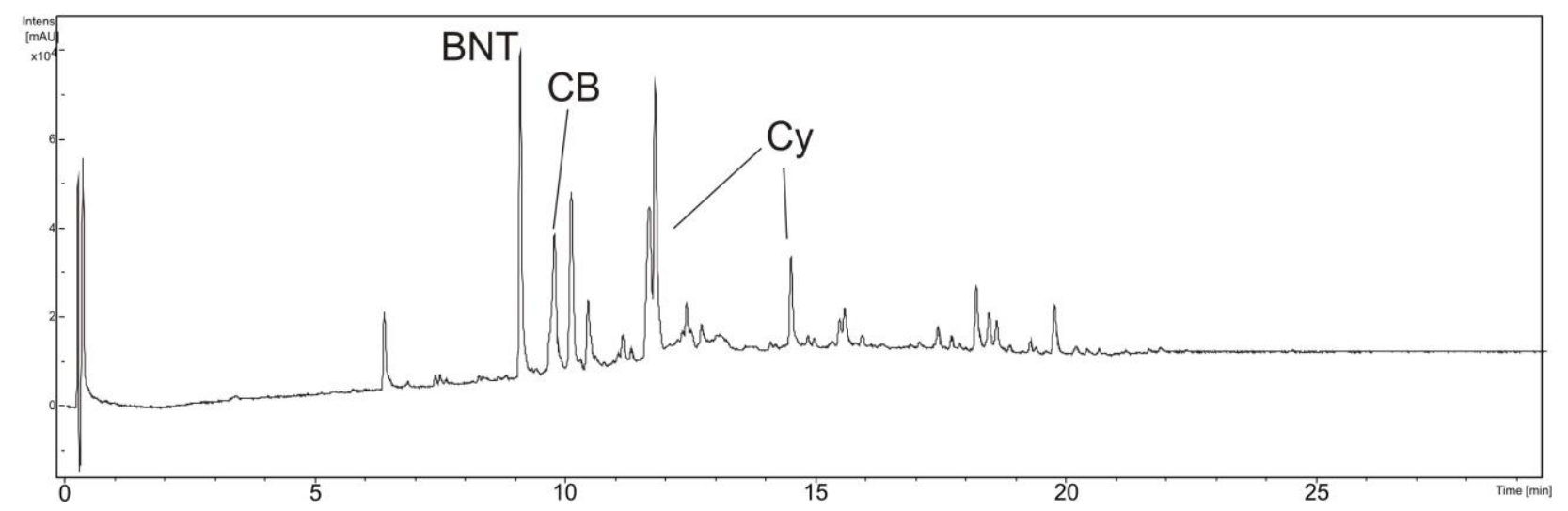

Fig. 5 - Stromatal HPLC-UV profiles of D. korfii. (Holotype) (BNT -binaphthalene tetrol; CB Concentricol B, Cy - cytochalasins).

\section{Acknowledgments}

The DAAD and the Argentine Ministerio de Ciencia, Tecnología e Innovación Productiva are gratefully acknowledged for an academic exchange grant. We would also like to express our gratitude to the Administration of National Parks of Argentina, the Ministry of the Environment of Salta Province and Provincial Direction of Biodiversity of Jujuy Province for authorization of collection.

\section{References}

Bills GF, González-Menéndez V, Martín J., Platas G et al. 2012 - Hypoxylon pulicicidum sp. nov. (Ascomycota, Xylariales), a pantropical insecticide-producing endophyte. PLoS ONE 7(10): e46687.

Bitzer J, Læssøe T, Fournier J, Kummer V et al. 2008 - Affinities of Phylacia and the daldinoid Xylariaceae, inferred from chemotypes of cultures and ribosomal DNA sequences. Mycological Research 112, 251-270.

Child M. 1932 - The genus Daldinia. Annals of the Missouri Botanical Garden 19, 429-496.

Guindon S, Dufayard JF, Lefort V, Anisimova M et al. 2010 - New algorithms and methods to estimate maximum-likelihood phylogenies: assessing the performance of PhyML 3.0. Systematic Biology 59, 307-321.

Hellwig V, Ju YM, Rogers JD, Fournier J, Stadler M. 2005 - Hypomiltin, a novel azaphilone from Hypoxylon hypomiltum, and chemotypes in Hypoxylon sect. Hypoxylon as inferred from analytical HPLC profiling. Mycological Progress 4, 39-54.

Hladki AI. 2004 - Contribución al estudio de las Xylariaceae de la República Argentina II). Los géneros Daldinia, Entonaema y Phylacia para Tucumán. Lilloa 41(1-2), 37-47. 
Hsieh HM, Ju YM, Rogers JD. 2005 - Molecular phylogeny of Hypoxylon and closely related genera. Mycologia 97, 844-865

Jayasiri SC, Hyde KD, Ariyawansa HA, Bhat J et al. 2015 - The Faces of Fungi database: fungal names linked with morphology, phylogeny and human impacts. Fungal Diversity 74:3-18.

Ju YM, Rogers JD, San Martín F. 1997 - A revision of the genus Daldinia. Mycotaxon 61, 243-293.

Ju YM, Rogers JD. 1996 - A revision of the genus Hypoxylon [Mycologia Memoir no. 20]. APS Press, St. Paul, MN.

Katoh K, Misawa K, Kuma K, Miyata T. 2002 - MAFFT: a novel method for rapid multiple sequence alignment based on fast Fourier transform. Nucleic Acids Research 30, 3059-3066

Kuhnert E, Heitkämper S, Fournier J, Surup F, Stadler M. 2014a - Hypoxyvermelhotins A-C, new pigments from Hypoxylon lechatii sp. nov. Fungal Biology 118, 242-252.

Kuhnert E, Fournier J, Peršoh D, Luangsa-ard JJ, Stadler M. 2014b - New Hypoxylon species from Martinique and new evidence on the molecular phylogeny of Hypoxylon based on ITS rDNA and $\beta$-tubulin data. Fungal Diversity 64, 181-203.

Kuhnert E, Surup F, Sir EB, Lambert C, et al. 2015 - Lenormandins A - G, new azaphilones from Hypoxylon lenormandii and Hypoxylon jaklitschii sp. nov., recognised by chemotaxonomic data. Fungal Diversity 71, 165-184

Kuhnert E, Sir EB, Lambert C, Hyde KD et al. 2017 - Phylogenetic and chemotaxonomic resolution of the genus Annulohypoxylon (Xylariaceae) including four new species. Fungal Diversity, in press (DOI 10.1007/s13225-016-0377-6).

Otto A, Laub A, Wendt L, Porzel A et al. 2016 - Chilenopeptins A and B, peptaibols from the Chilean Sepedonium aff. chalcipori KSH 883. Journal of Natural Products 79, 929-938.

Pažoutová S, Follert S, Bitzer J, Keck M et al. 2013- A new endophytic insect-associated Daldinia species, recognised from a comparison of secondary metabolite profiles and molecular phylogeny. Fungal Diversity 60, 107-123.

Quang DN, Hashimoto T, Tanaka M, Baumgartner M et al. 2002 - Concentricols B, C and D, three novel squalene-type triterpenoids from the ascomycete Daldinia concentrica. Phytochemistry 61, 345-353.

Rayner RW. 1970 - A mycological colour chart. Commonwealth Mycological Institute, Kew and British Mycological Society.

Sir EB, Kuhnert E, Lambert C, Hladki AI et al. 2016 - New species and reports of Hypoxylon from Argentina recognized by a polyphasic approach. Mycological Progress 15, 42.

Sir EB, Kuhnert E, Surup F, Hyde KD, Stadler M. 2015 - Discovery of new mitorubrin derivatives from Hypoxylon fulvo-sulphureum sp. nov. (Ascomycota, Xylariales). Mycological Progress 14,28

Spegazzini CL. 1902 - Mycetes Argentinenses. Anales del Museo Nacional de Historia Natural de Buenos Aires 8 (1), 51-190.

Spegazzini CL. 1909 - Mycetes Argentinenses. Anales del Museo Nacional de Historia Natural de Buenos Aires 19 (12), 257-458.

Stadler M, Hawksworth DL, Fournier J. 2014a - The application of the name Xylaria hypoxylon, based on Clavaria hypoxylon of Linnaeus. IMA Fungus 5, 57-66.

Stadler M, Læssøe T, Fournier J, Decock C et al. 2014b - A polyphasic taxonomy of Daldinia (Xylariaceae). Studies in Mycology 77, 1-143.

Stadler M, Wollweber H, Mühlbauer A, Henkel T et al. 2001 - Secondary metabolite profiles, genetic fingerprints and taxonomy of Daldinia and allies. Mycotaxon 77, 379-429.

Stamatakis A. 2014 - RAxML Version 8: A tool for phylogenetic analysis and post-analysis of large phylogenies. Bioinformatics 30, 1312-1313.

Talavera G, Castresana J. 2007 - Improvement of phylogenies after removing divergent and ambiguously aligned blocks from protein sequence alignments. Systematic Biology 56, 564577.

Tamura K, Stecher G, Peterson D, Filipski A, Kumar S. 2013 - MEGA6: Molecular Evolutionary Genetics Analysis Version 6.0. Molecular Biology and Evolution 30, 2725-2729. 
Triebel D, Peršoh D, Wollweber H, Stadler M. 2005 - Phylogenetic relationships among Daldinia, Entonaema and Hypoxylon as inferred from ITS nrDNA sequences. Nova Hedwigia 80, 2543. 